\title{
Study on the Effect of Microwave Irradiation on Rock Strength
}

\author{
Qin Like $^{1, *}$, Dai Jun ${ }^{1}$ and Teng Pengfei ${ }^{2}$ \\ 1. School of Architecture and Civil Engineering, Xi'an University of Science and Technology, Xi'an 710054, China \\ 2. University of Hong Kong, 10 Sassoon Road, Pok Fu Lam, Hong Kong
}

Received 28 January 2015; Accepted 13 June 2015

\begin{abstract}
Microwave-assisted rock breaking can effectively improve rock breaking efficiency, decrease energy consumption, and promote the development of miniaturized and light-weight rock breaking equipment. This study uses rock mass with structural surface as the research object and analyzes the representative volume element of rock mass at varied temperatures. The general expression of rock mass strength is first obtained. The analytical formula of rock mass strength under microwave irradiation is then derived on the basis of temperature rise features under microwave irradiation. The analytical formula is verified by the experiment. Finally, the variation law of rock mass strength is studied. Results show that microwave irradiation effectively decreases rock mass strength. A longer microwave irradiation time corresponds to high microwave power density and low rock mass strength. Rock mass strength decreases linearly when microwave irradiation time and microwave power density increases. The reduction of the rock structural surface is greater than the rock mass strength under the same microwave irradiation.
\end{abstract}

Keywords: Microwave-assisted rock breaking; Rock mass strength; Irradiation time; Power density

\section{Introduction}

A microwave is an electromagnetic wave with a wavelength of $1 \mathrm{~mm}$ to $1 \mathrm{~m}$ and is considered an ultra-high frequency electromagnetic wave. Some advantages of microwave heating over conventional heating include internal heating, rapid heating, selective heating, and ease-of-control. Moreover, microwave heating is widely used in food, agriculture, medicine, and metallurgy [1-5]. Microwave heating can also be used in auxiliary rock breaking. Microwave-assisted rock breaking decreases rock mass strength by microwave irradiation and then by mechanical and hydraulic breaking. Microwave-assisted rock breaking effectively improves rock breaking efficiency, increases the speed of excavation driving, and decreases energy consumption. Moreover, microwave-assisted rock breaking is widely used in tunnel excavation, roadway driving, and mineral crushing and sorting [6]. Furthermore, given the development of space technology, this method can be used in the mining of other celestial bodies in the future. Microwave-assisted rock breaking promotes the development of miniature and light-weight rock breaking equipment, thus decreasing interstellar transport cost and allowing operations in different gravity field environments. Thus, microwave-assisted rock breaking is the method of choice for mining in outer space [7].

This research mainly focuses on comparing rock strength before and after microwave irradiation by conducting experiments and analyzing rock damage mechanism and impacting factors in the microwave field by the numerical method. Hassani [8] conducted a microwave irradiation

* E-mail address: 422576294@qq.com ISSN: 1791-2377 @ 2015 Kavala Institute of Technology. All rights reserved. experiment on basalt from America, Canada, and China and compared rock strength before and after microwave irradiation. The results showed a reduction of tensile strength and compressive strength under the microwave irritation. The range also increased with increasing microwave power and irradiation time. Jun Dai [9] conducted an irradiation experiment on granite from Xi'an. The experiment showed that the tensile strength of granite decreased substantially under microwave irradiation. The tensile strength of some granite also decreased by $40 \%$, thus indicating that microwave pre-treatment decreases rock strength efficiency and contributes to rock breaking. Whittles [10] analyzed the strength of gangue consisting of calcite and pyrite in the microwave field by using the finite difference method from a microscopic view and considered the influence of microwave power and irradiation time on rock strength. Jones [11] studied the particles of pyrite and calcite. The results show a reduction of rock strength with increasing microwave power and irradiation time. Jones [12] analyzed the rock particles of pyrite and calcite and rock breaking types under microwave irradiation from a microscopic view. Yicai Wang [13] analyzed a single particle of pyrite and calcite by the numerical method and studied the stress distribution and microcrack development inside a rock particle from a microscopic view. Jun Dai [14] simulated a rock damage mechanism under microwave irradiation by using the numerical method from a microscopic view. Yan Zhang [15] conducted a theoretical analysis of the influence of temperature stress on the strength of fractured rock mass. At present, few studies have reported the change of rock mass strength under varied temperature, particularly under microwave irradiation.

The experiment proved that microwave irradiation decreased rock strength and explained the mechanism damage by numerical method from a microscopic view. However, the microscopic scale of the theoretical study 
deviated from macro conditions, thus restricting further research on microwave-assisted rock breaking. The present paper uses the representative volume element (RVE) of rock mass with structural surface as the research object. First, the expression of the strength of the rock mass under the general temperature variation of rock mass RVE is deduced. Second, the analytical formula of the rock mass strength in the microwave field is derived. Finally, the accuracy of the formula is verified by the numerical method.

\section{Effect of temperature variation on rock strength}

2.1 Fundamental solution of the thermal elastic plane strain problem

The physical equation of the plane strain when the temperature changes can be expressed as follows:

$$
\left.\begin{array}{l}
\left.\varepsilon_{x}=\frac{1-\mu^{2}}{E}\left[\sigma_{x}-\frac{\mu}{1-\mu} \sigma_{y}\right)\right]+(1+\alpha) \Delta T \\
\left.\varepsilon_{y}=\frac{1-\mu^{2}}{E}\left[\sigma_{y}-\frac{\mu}{1-\mu} \sigma_{x}\right)\right]+(1+\alpha) \Delta T \\
\gamma_{x y}=\frac{1}{G} \tau_{x y}
\end{array}\right\}
$$

Where, $\Delta T$ is the temperature variation of rock mass, $\alpha$ is the thermal expansion coefficient of the rock mass, $\mathrm{E}$ is Young's modulus, $\mu$ is Poisson's ratio, and $G$ is the shear modulus.

Equilibrium equation:

$$
\left.\begin{array}{c}
\frac{\partial \sigma_{x}}{\partial x}+\frac{\partial \tau_{y x}}{\partial y}=0 \\
\frac{\partial \sigma_{y}}{\partial y}+\frac{\partial \tau_{x y}}{\partial x}=0
\end{array}\right\}
$$

The stress can be expressed by transforming Formula (1):

$$
\left.\begin{array}{l}
\sigma_{x}=\tilde{\sigma}_{x}-\frac{E}{1-2 \mu} \alpha \Delta T \\
\sigma_{y}=\tilde{\sigma}_{y}-\frac{E}{1-2 \mu} \alpha \Delta T \\
\tau_{x y}=\tilde{\tau}_{x y}
\end{array}\right\}
$$

where,

$$
\begin{aligned}
& \tilde{\sigma}_{x}=\frac{E}{(1-\mu)(1-2 \mu)}\left(\varepsilon_{x}+\frac{\mu}{1-\mu} \varepsilon_{y}\right), \\
& \tilde{\sigma}_{y}=\frac{E}{(1-\mu)(1-2 \mu)}\left(\varepsilon_{y}+\frac{\mu}{1-\mu} \varepsilon_{x}\right) .
\end{aligned}
$$

The following formula can be obtained when Type (3) is substituted into the equilibrium in Equation (2):

$$
\left.\begin{array}{c}
\frac{\partial \tilde{\sigma}_{x}}{\partial x}+\frac{\partial \tilde{\tau}_{y x}}{\partial y}-\frac{E \alpha}{1-2 \mu} \frac{\partial T}{\partial x}=0 \\
\frac{\partial \tilde{\sigma}_{y}}{\partial y}+\frac{\partial \tilde{\tau}_{x y}}{\partial x}-\frac{E \alpha}{1-2 \mu} \frac{\partial T}{\partial y}=0
\end{array}\right\}
$$

The following formula can be obtained when Type (3) is substituted into boundary conditions:

$$
\left.\begin{array}{c}
\tilde{\sigma}_{x} l+\tilde{\tau}_{y x} m=\frac{E \alpha \Delta T}{1-2 \mu} l \\
\tilde{\tau}_{x y} l+\tilde{\sigma}_{y} m=\frac{E \alpha \Delta T}{1-2 \mu} m
\end{array}\right\}
$$

Formulas (4) and (5) show that the displacement in the elastic rock mass produced by temperature change can be equivalent to the stress produced by the imaginary volume force $\frac{E \alpha}{1-2 \mu} \frac{\partial T}{\partial x}$ and $\frac{E \alpha}{1-2 \mu} \frac{\partial T}{\partial y}$ and the imaginary surface force $\frac{E \alpha \Delta T}{1-2 \mu} l$ and $\frac{E \alpha \Delta T}{1-2 \mu} m$ under consistent temperature. $\tilde{\sigma}_{x}$ and $\tilde{\sigma}_{y}$ are calculated by the displacement method or stress method, and the stress under temperature change is obtained by substituting the aforementioned variables into Formula (3).

\subsection{Effect of temperature variation on rock mass strength}

This calculation assumes that rock mass is a linear elastic body and is in compressive status before and after temperature change, the rock block is isotropic, and the strength of the rock mass and structural surface follow the Mohr-Coulomb criterion.

The RVE unit is shown in Figure 1: (a) the stress state at the initial temperature, (b) the stress state caused by the temperature change, and (c) the total stress state after temperature change. Figure 1 shows that a structural surface $\mathrm{AB}$ develops in rock mass. The normal stress $\sigma$ and shearing stress $\tau$ on plane AB can be obtained by Mohr's circle if $\beta$ is assumed as the included angle between plane $A B$ and the minimum principal stress direction. The calculation can be expressed as follows:

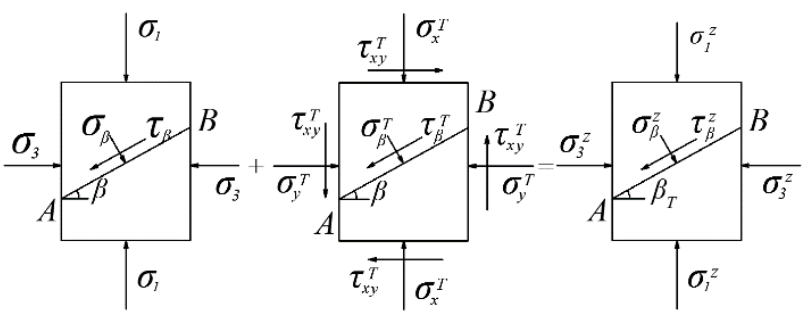

(a)

(b)

(c)

Fig. 1. Calculation mode of RVE

$$
\left.\begin{array}{l}
\sigma_{\beta}=\frac{1}{2}\left(\sigma_{1}+\sigma_{3}\right)-\frac{1}{2}\left(\sigma_{1}-\sigma_{3}\right) \cos 2 \beta \\
\tau_{\beta}=\frac{1}{2}\left(\sigma_{1}-\sigma_{3}\right) \sin 2 \beta
\end{array}\right\}
$$


The stress on the structural surface when the temperature of rock mass changes is expressed as follows:

$$
\begin{aligned}
& \sigma_{\beta}^{T}=\frac{1}{2}\left(\tilde{\sigma}_{x}+\tilde{\sigma}_{y}-2 \frac{E}{1-2 \mu} \alpha \Delta T\right) \\
& +\frac{1}{2}\left(\tilde{\sigma}_{x}-\tilde{\sigma}_{y}\right) \cos 2 \beta-\tilde{\tau}_{x y} \sin 2 \beta \\
& \tau_{\beta}^{T}=\frac{1}{2}\left(\tilde{\sigma}_{x}-\tilde{\sigma}_{y}\right) \sin 2 \beta+\tilde{\tau}_{x y} \cos 2 \beta
\end{aligned}
$$

The total stress on the structural surface when considering the temperature variation is expressed as follows:

$$
\left.\begin{array}{l}
\sigma_{\beta}^{z}=\frac{1}{2}\left(\sigma_{1}+\sigma_{3}\right)-\frac{1}{2}\left(\sigma_{1}-\sigma_{3}\right) \cos 2 \beta+\sigma_{\beta}^{T} \\
\tau_{\beta}^{z}=\frac{1}{2}\left(\sigma_{1}-\sigma_{3}\right) \sin 2 \beta+\tau_{\beta}^{T}
\end{array}\right\}
$$

The structural surface strength is subject to the MohrCoulomb criterion.

$$
\tau_{\beta}^{z}=c_{j}+\sigma_{\beta}^{z} \tan \phi_{j},
$$

where $c_{j}$ and $\varphi_{j}$ are structural surface bonding strength and internal friction angle, respectively.

The following formula can be obtained when Formula (8) is substituted into Formula (9):

$$
\sigma_{1}=\sigma_{3} \cot ^{2} \beta \frac{\tan \beta+\tan \phi_{j}}{\cot \beta-\tan \phi_{j}}+\frac{\sigma_{\beta}^{T} \tan \phi_{j}-\tau_{\beta}^{T}+c_{j}}{\sin ^{2} \beta\left(\cot \beta-\tan \phi_{j}\right)}
$$

The structural surface stress is in limit equilibrium state when stress satisfies the Formula (10).

Rock mass strength is determined by the strength of the rock block when the rock mass is not destroyed along the structural surface. The principal stress of rock mass can be expressed as follows:

$\sigma_{1}^{T}=\frac{\sigma_{1}+\sigma_{3}+\sigma_{x}^{T}+\sigma_{y}^{T}}{2} \pm \sqrt{\left[\frac{\sigma_{1}-\sigma_{3}+\sigma_{x}^{T}-\sigma_{y}^{T}}{2}\right]^{2}-\tau_{x y}^{T 2}}$

The angle between the structural surface and small principal stress changes because of the existence of temperature stress. The value after the change is expressed as follows:

$$
\beta_{T}=\beta-\frac{\pi}{2}+\frac{1}{2} \operatorname{arcsian}\left(\frac{2 \tau_{x y}^{T}}{\sigma_{1}-\sigma_{3}+\sigma_{x}^{T}-\sigma_{y}^{T}}\right)
$$

The rock mass is in a critical state when the principal stress of the rock mass under temperature change satisfies the Mohr-Coulomb criterion.

$$
\sigma_{1}^{T}=\sigma_{3}^{T} \tan ^{2}\left(45^{\circ}+\frac{\phi}{2}\right)+2 c \tan \left(45^{\circ}+\frac{\phi}{2}\right)
$$

The rock block strength can be obtained from Formulas (11) and (13).

The rock mass strength after temperature change can be expressed by Figure 2, which shows that the rock mass will be destroyed along the structural surface when $\beta_{1} \leq \beta_{T} \leq \beta_{2}$. The rock mass strength is calculated by Formula (10). The rock mass will not be destroyed along the structural surface when $\beta_{T}<\beta_{1}$ or when $\beta_{T}>\beta_{2}$. Moreover, the rock mass strength is determined by the strength of the rock block. The rock mass strength is obtained from Formulas (11) and (13).

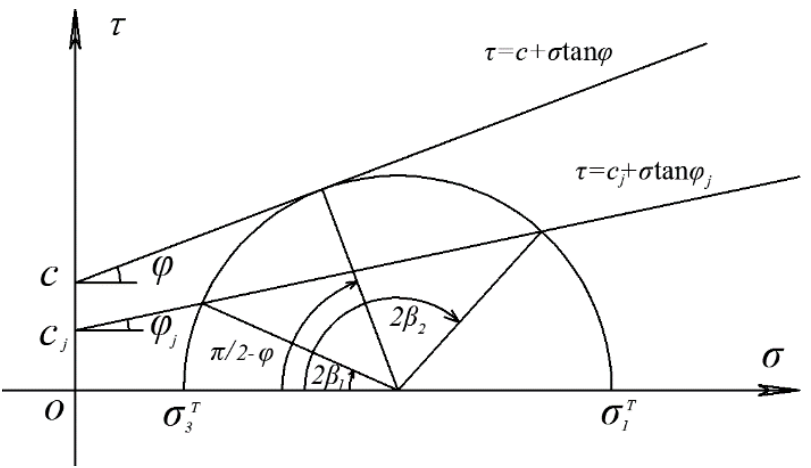

Fig. 2. Strength schematic of a single structural surface rock mass

The geometrical relationship in Figure 2 shows that the values of $\beta_{1}$ and $\beta_{2}$ can be determined by the following formula:

$$
\beta_{1}=\frac{1}{2} \arcsin \frac{\left(\sigma_{1}^{T}+\sigma_{3}^{T}+2 c_{j} \cot \phi_{j}\right) \sin \phi_{j}}{\sigma_{1}^{T}-\sigma_{3}^{T}}-\frac{\phi_{j}}{2}
$$

$$
\beta_{2}=\frac{\pi}{2}-\frac{1}{2} \arcsin \frac{\left(\sigma_{1}^{T}+\sigma_{3}^{T}+2 c_{j} \cot \phi_{j}\right) \sin \phi_{j}}{\sigma_{1}^{T}-\sigma_{3}^{T}}-\frac{\phi_{j}}{2}
$$

If the rock mass contains two or more groups of structural surface, the rock mass strength can be determined by single structural surface theory. The strength envelope and Mohr's circle of stress is obtained when each group of structural surface exists. $\sigma_{3}^{T}$ and the angle between each group of structural surface determine the structural surface group of the rock that will be destroyed. The rock mass strength is determined by the strength of the structural surface.

\section{Effect of microwave irradiation on rock mass strength}

The heat energy produced by microwave irradiation is mainly dependent on the frequency and intensity of the electric field. The heat generated by the material of unit volume can be calculated by the following formula ${ }^{[7-11]}$.

$P_{d}=2 \pi f \varepsilon_{o} \varepsilon_{r}^{\prime \prime} E_{o}^{2}$

Where, $P_{d}$ is the microwave power density $\left(W / \mathrm{m}^{3}\right)$, i.e., the microwave power that converts into heat energy; $f$ is the 
microwave frequency $(\mathrm{Hz}) ; \mathcal{E}_{o}$ is the vacuum dielectric coefficient $\left(8.854 \times 10^{-12} \mathrm{~F} / \mathrm{m}\right) ; \varepsilon_{r}^{\prime \prime}$ is the dielectric loss factor of the rock mass; $E_{\mathrm{o}}$ is the electric field valid value $(\mathrm{V} / \mathrm{m})$.

The temperature increase of the RVE element in the microwave field is uniform because microwave heating involves the uniform heating of the body. The increase of temperature can be expressed as follows:

$$
\Delta T=\frac{P_{d} t}{\rho C}
$$

Where, $t$ is the microwave irradiation time (s), $\rho$ is the rock mass density $\left(\mathrm{kg} / \mathrm{m}^{3}\right)$, and $C$ is the specific heat $(\mathrm{J} / \mathrm{kg}, \mathrm{K})$.

The thermal stress generated by a rock under microwave irradiation can be obtained by substituting Formula (17) Formulas (1) to (5):

$$
\left.\begin{array}{l}
\sigma_{x}^{T}=-\frac{\alpha E P_{d} t}{(1-2 \mu) \rho C} \\
\sigma_{y}^{T}=-\frac{\alpha E P_{d} t}{(1-2 \mu) \rho C} \\
\tau_{x y}^{T}=0
\end{array}\right\}
$$

The strength of the rock structural surface under microwave irradiation when Formula (18) is substituted into Formula (10) can be expressed as follows:

$$
\begin{aligned}
\sigma_{1}=\sigma_{3} \cot ^{2} \beta \frac{\tan \phi_{j}+\tan \beta}{\cot \beta-\tan \phi_{j}}+ \\
\frac{c_{j}(1-2 \mu) \rho C-\alpha t E P_{d} \tan \phi_{j}}{\sin ^{2} \beta\left(\cot \beta-\tan \phi_{j}\right)(1-2 \mu) \rho C}
\end{aligned}
$$

When Formula (17) is substituted into Formulas (13) and (14), the rock strength under microwave irradiation can be expressed as follows:

$$
\begin{array}{r}
\sigma_{1}=\sigma_{3} \tan ^{2}\left(45^{\circ}+\frac{\phi}{2}\right)+2 c \tan \left(45^{\circ}+\frac{\phi}{2}\right) \\
-\frac{\alpha E P_{d} t}{(1-2 \mu) \rho C}\left[\tan ^{2}\left(45^{\circ}+\frac{\phi}{2}\right)-1\right]
\end{array}
$$

Mohr's stress circle of RVE element does not change in size under microwave irradiation. However, the position of Mohr's circle shifts along the axis of $\sigma$. The displacement value is $\frac{\alpha E P_{d} t}{(1-2 \mu) \rho C}$. The displacement leads to two results: (1) the strength of rock mass decreases whether the rock is broken along the structural surface or the rock block; (2) the $\beta$ scale along the structural surface increases with increasing $\left(\beta_{2}-\beta_{1}\right)$.

The strength of rock mass with multiple groups of structures under microwave irradiation can be obtained by using single structural surface theory.

\section{Experimental verification and influencing factor analysis}

A microwave irradiation experiment is made on the granite to verify the rationality of the formula. The microwave irradiation device is shown in Figure 3 and has three parts: microwave apparatus, waveguide, and furnace chamber. The test is performed only on the intact rock mass without structural surface because of the difficulty of making rock samples with a structural surface. The basic physical parameters of granite are as follows: density $\rho=2500 \mathrm{~kg} / \mathrm{m}^{3}$, rock cohesion $c=25 \mathrm{MPa}$, rock block friction angle $\varphi=45^{\circ}$, elastic modulus $E=3.5 \times 10^{4} \mathrm{MPa}$, Poisson's ratio $\mu=0.25$, thermal expansion coefficient $\alpha=7.08 \times 10^{-6}$, and specific heat $C=780 \mathrm{~J} /\left(\mathrm{kg} \cdot{ }^{\circ} \mathrm{C}\right)$.

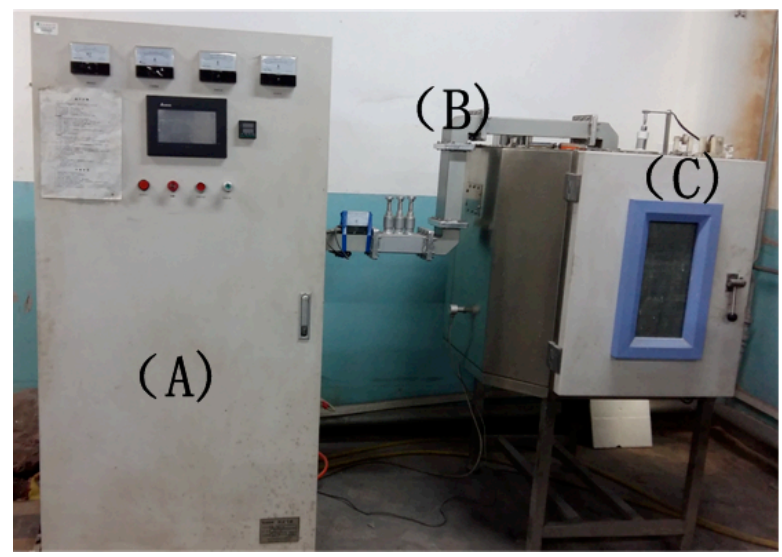

(A) Microwave apparatus (B) Waveguide (C) Furnace chamber Fig. 3. Microwave irradiation equipment

The granite sample irradiated by the microwave is shown in Figure 4. This figure also shows that microwave irradiation causes a number of cracks in the surface of granite samples. A main crack is horizontally distributed and many secondary cracks are vertically distributed. Figure 5 compares the experimental values and calculated values of rock samples under different microwave irradiation time. Both the calculated and experimental values decrease with increasing irradiation time. Their numerical values are close, and the biggest difference is $8 \%$ only.
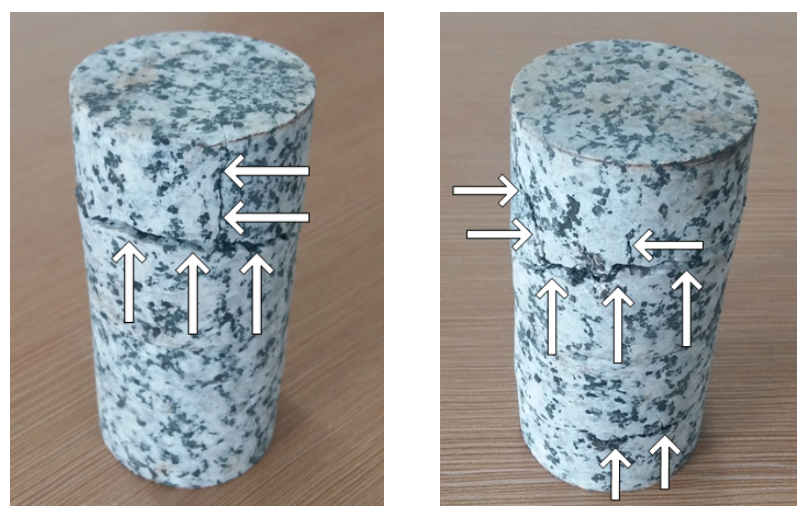

Fig. 4. A granite sample irradiated by microwave 


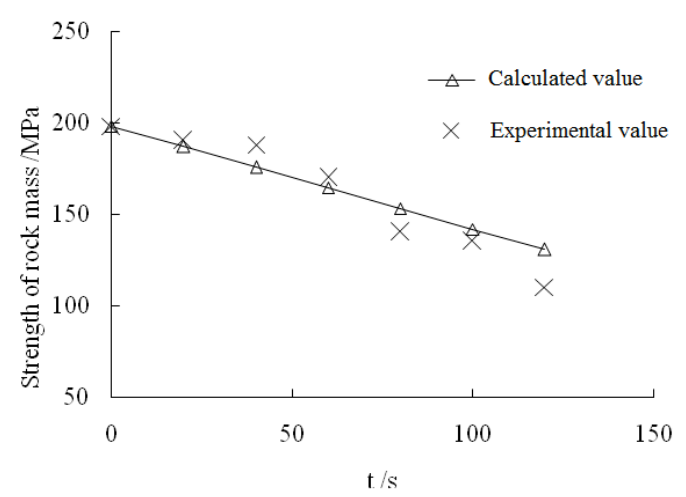

Fig. 5. Comparison of the experimental and calculated values of rock mass strength.

Figure 6 shows the relationship between rock mass strength and structural surface angle when the power density is $\mathrm{P}_{\mathrm{d}}=8 \times 10^{6} \mathrm{~W} / \mathrm{m}^{3}$ and irradiation time is $\mathrm{t}=0,30$, and 60 s. The curve segment is the result of rock breaking along the structural surface, and the rock mass strength is determined by the strength of the structural surface. The horizontal segment is the result of rock breaking in a surface other than along the structural surface, and rock mass strength is determined by rock block strength. The figure shows the reduction of both rock structural strength and rock block strength under microwave irradiation. Rock mass strength decreases with increasing irradiation time.

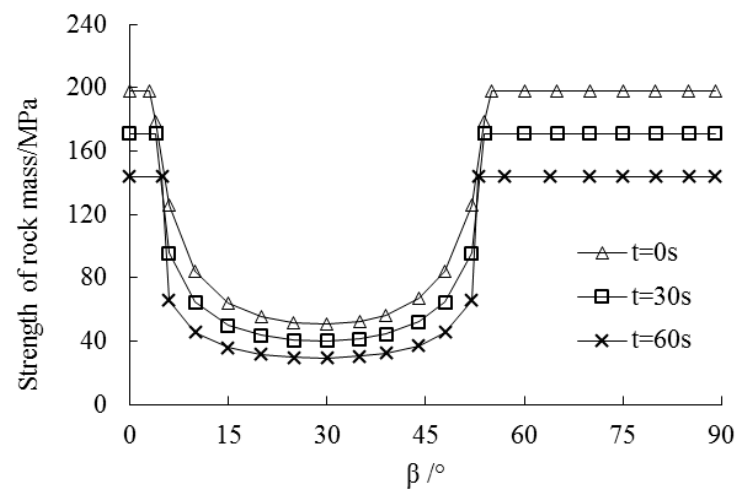

Fig. 6. Relationship between rock mass strength and structural surface angle

Figure 7 shows the relationship between rock mass strength and microwave irradiation time under the different dip angles of the structural surface. Figure 7 shows the relationship between rock mass and microwave power density under the different dip angles of the structural surface. The rock mass strength decreases when irradiation time and power density increase regardless of the size of the dip angle of the structure. The rock mass strength is linearly related to microwave irradiation time and microwave power density. Microwave irradiation time changes from $0 \mathrm{~s}$ to 60 $\mathrm{s}$, and the structural surface angle $\beta$ is $30^{\circ}$ when $\mathrm{P}_{\mathrm{d}}=8 \times 10^{6}$ $\mathrm{W} / \mathrm{m}^{3}$. Rock mass is destroyed along the structural surface. Thus, rock strength is controlled by the structural surface. Rock mass strength also decreases from 50.6 MPa to 29.6 $\mathrm{MPa}$, with a decrease in percentage of $41.5 \%$. When the structural dip angle is $60^{\circ}$, the rock mass strength is determined by rock block strength and decreases from 198.1 $\mathrm{MPa}$ to $144.2 \mathrm{MPa}$, with a decrease percentage of $27.2 \%$. When the irradiation time is $\mathrm{t}=60 \mathrm{~s}$, power density increases from $2 \times 10^{6} \mathrm{~W} / \mathrm{m}^{3}$ to $8 \times 10^{6} \mathrm{~W} / \mathrm{m}^{3}$, the structural surface dip angle $\beta$ is $30^{\circ}$ and rock mass strength decreases from $45.4 \mathrm{MPa}$ to $29.6 \mathrm{MPa}$, with a decrease percentage of $44.5 \%$. Thus, when the dip angle of the structural surface $\beta$ is $60^{\circ}$, the rock mass strength decreases from $184.6 \mathrm{MPa}$ to 144.2 $\mathrm{MPa}$, with a decrease percentage of $21.2 \%$. The strength of the rock mass structure decreases more than that of the rock block under different microwave irradiation conditions. The reductions indicate the significant influence of microwave irradiation on the strength of the structural surface.

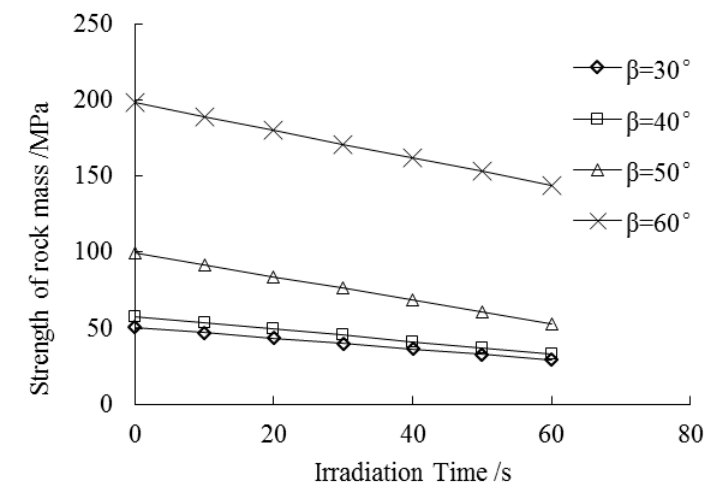

Fig. 7. Relationship between rock mass strength and microwave irradiation time

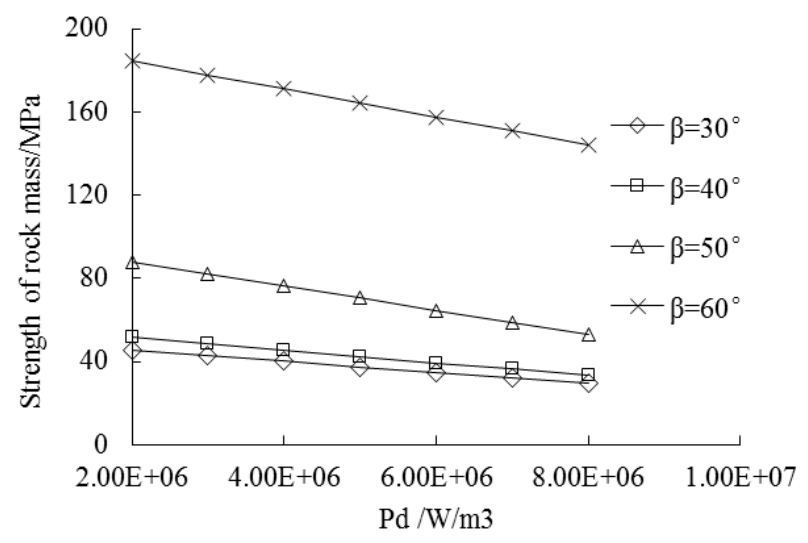

Fig. 8. Relationship between rock mass strength and power density

\section{Conclusions}

This paper uses a rock mass with structural surface as the object of study, derives the general expression of rock mass strength under temperature change by using the elastic superposition principle, and obtains the analytical formula of rock mass strength under microwave irradiation on the basis of the temperature rise features under microwave irradiation. The analytical formula is verified by the experiment. The research results show the following. Microwave irradiation effectively reduces rock mass strength, and a longer microwave irradiation time corresponds to higher microwave power density and lower rock mass strength. Rock mass strength decreases linearly with increasing microwave irradiation time and microwave power density. The rock structural surface strength decreases more than rock mass strength under the same microwave irradiation.

Factors, such as the angle of the rock structural surface, microwave irradiation power, and irradiation time, have been considered in the formula derivation. This formula can be used to predict rock mass strength and can provide a 
scientific foundation for microwave-assisted rock breaking, microwave source selection, and microwave equipment optimization and design.

\section{References}

1. Islam, Mohammad Moinul. "Design of a microstrip antenna on duroid 5870 substrate material for $\mathrm{ku}$ and k-band applications", Tehnicki Vjesnik, 22 (1), 2015, pp. 71-77.

2. Lubikowski, Kamil. "Seebeck phenomenon, calculation method comparison", Journal of Power Technologies, 95, 2015, pp. 63-67.

3. Czaplicka-Kolarz, Krystyna-Burchart-Korol, et al. "Model of ecoefficiency assessment of mining production processes", Archives of Mining Sciences, 1 (2), 2015, pp. 477-482.

4. Lindroth D P; Berglund, W R., "Microwave assisted drilling in hard rock", Tunnels \& Tunnelling, 1(12), pp. 54-59.

5. S.W. Kingman, K. Jackson, "Recent developments in microwaveassisted combination", International Journal of Mineral Processing, 74(2), 2004, pp. 71-83.

6. Ferri Hassani, Pejman Nekoovaght, "The development of microwave assisted machineries to break hard rocks", Seoul, Korea: Proceedings of the 28th ISARC, 2011, pp. 678-684.

7. Hemanth Satish, "Exploring microwave assisted rock breakage for possible space mining applications", Montreal: McGill University. 2005. pp. 25-32

8. F. Hassani, P. M. Nekoovaght, P. Radziszewski, et al. "Microwave assisted mechanical rock breaking", Harmonising Rock Engineering and the Environment, London: Taylor \& Francis Group, 2011, pp. 57-60.

9. Jun Dai, Zhen Meng, Bingquan Wu, "Study on Impact of Rock Strength by Microwave Irradiation", Nonferrous Metals, (3), 2014, pp. 32-36.
10. D.N. Whittles, S.W. Kingman, D.J. Reddish. "Application of numerical modeling for prediction of the influence of Power Density on Microwave-Assisted Breakage", International Journal of Mineral Processing, 68 (4), 2003, pp. 71-91.

11. D.A. Jones, S.W. Kingman, D.N. Whittles, I.S. Lowndes, "The influence of microwave energy delivery method on strength reduction in ore samples", Chemical Engineering and Processing, 46 (4), 2007, pp. 291-299.

12. D.A. Jones, S.W. Kingman, D.N. Whittles, et al. "Understanding microwave assisted breakage", Minerals Engineering, 18 (7), 2005, pp. 659-669.

13. Yicai Wang, Nenad Djordjevic, "Thermal stress FEM analysis of rock with microwave energy", International Journal of Mineral Processing, 130, 2014, pp. 82-89.

14. Jun Dai, Like Qin, "The stimulation analysis of the rock damage under the microwave irradiation from microscopic view", Journal of $X i$ 'an University of Science and Technology, 34 (6), 2014, pp. 652655.

15. Yan Zhang, Ning Li, Haiming Yu, et al. "Study on the impact of temperature stress to the rock mass strength", Chinese Journal of Rock Mechanics and Engineering, 32(s1), 2013, pp. 2660-2668. 